J. Korean Math. Soc. 50 (2013), No. 2, pp. 445-464

http://dx.doi.org/10.4134/JKMS.2013.50.2.445

\title{
THE CUSP STRUCTURE OF THE PARAMODULAR GROUPS FOR DEGREE TWO
}

\author{
Cris Poor And David S. Yuen
}

\begin{abstract}
We describe the one-dimensional and zero-dimensional cusps of the Satake compactification for the paramodular groups in degree two for arbitrary levels. We determine the crossings of the one-dimensional cusps. Applications to computing the dimensions of Siegel modular forms are given.
\end{abstract}

\section{Introduction}

Paramodular groups arise naturally in the classification of polarized abelian varieties. The orbits of the Siegel upper half space under the action of a paramodular group constitute the moduli space of equivalence classes of abelian varieties with a fixed type of polarization. This interpretation of the paramodular groups and their automorphic forms played an important role in the articles of Siegel and Christian and in the textbook of Igusa (see $[3,13,19])$. More recently, the degree two case has become of particular interest. Roberts and Schmidt have given a systematic theory of newforms [15] for paramodular groups of degree two. Brumer and Kramer have proposed the Paramodular Conjecture [2], a testable modularity conjecture relating certain abelian surfaces of conductor $N$ defined over $\mathbb{Q}$ to certain weight two Hecke eigenforms for the degree two paramodular group of level $N$. This recent interest in degree two may have more to do with the identity of a paramodular group as an orthogonal group rather than as a symplectic group (see [7]). In order to maintain contact with the large body of techniques for computing with Siegel modular forms however, it is still important to study degree two paramodular groups as symplectic groups.

In this article we determine the structure of the Satake compactification for each paramodular group of degree two. That is, we completely classify the 1 -cusps and the 0 -cusps as well as the incidence relations among them. The double cosets corresponding to the 1-cusps were already classified by Reefschläger in his thesis [14]. The result is very nice: for level $N$, there is one double

Received July 24, 2012

2010 Mathematics Subject Classification. 11F46.

Key words and phrases. paramodular, Satake compactification. 


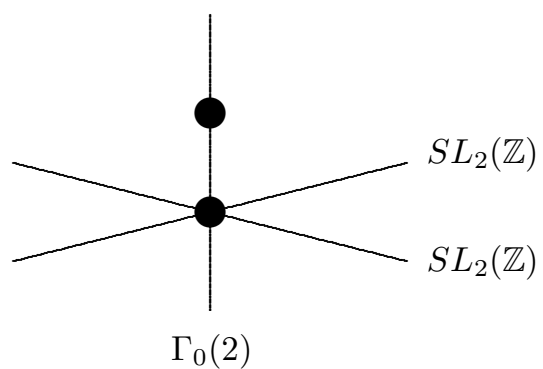

Figure 1. The cusp structure of $K(4)$.

coset for each positive integral divisor of $N$. Due to a theorem of Satake [18], possessing the cusp structure has the immediate application of determining the codimension of the cusp forms inside the space of modular forms for even weights $k>4$. We give two illustrations, made possible by our results here, of Satake's theorem. Let the degree two paramodular group of level $N$ be given by

$$
K(N)=\left\{\left(\begin{array}{cccc}
* & N * & * & * \\
* & * & * & * / N \\
* & N * & * & * \\
N * & N * & N * & *
\end{array}\right): * \in \mathbb{Z}\right\} \cap S p_{2}(\mathbb{Q}) .
$$

Thus, elements of $K(N)$ are characterized as rational symplectic matrices with certain divisibility properties. Let $M_{k}(K(N))$ and $S_{k}(K(N))$ be the Siegel modular forms and cusp forms, respectively, automorphic with respect to $K(N)$. For $N=4$, the three 1 -cusps cross as in Figure 1 and for even $k>4$, we have $\operatorname{dim} M_{k}(K(4))-\operatorname{dim} S_{k}(K(4))=2+2 \operatorname{dim} S_{k}\left(\mathrm{SL}_{2}(\mathbb{Z})\right)+\operatorname{dim} S_{k}\left(\Gamma_{0}(2)\right)$. In [12], the generating function of the modular forms for $K(4)$ was given:

$$
\begin{aligned}
& \sum_{k=0}^{\infty} \operatorname{dim} M_{k}(K(4)) t^{k} \\
= & \frac{\left(1+t^{12}\right)\left(1+t^{6}+t^{7}+t^{8}+t^{9}+t^{10}+t^{11}+t^{17}\right)}{\left(1-t^{4}\right)^{2}\left(1-t^{6}\right)\left(1-t^{12}\right)} \\
= & 1+2 t^{4}+2 t^{6}+t^{7}+4 t^{8}+t^{9}+5 t^{10}+3 t^{11}+10 t^{12}+3 t^{13}+\cdots .
\end{aligned}
$$

The cusp structure of $K(4)$ and an application of Satake's Theorem yield the following new result.

Theorem 1.1. We have

$$
\begin{aligned}
& \sum_{k=0}^{\infty} \operatorname{dim} S_{k}(K(4)) t^{k} \\
= & \frac{t^{7}+t^{8}+t^{9}+2 t^{10}+t^{11}+2 t^{12}+t^{16}+t^{17}+2 t^{18}+t^{19}+t^{20}+t^{21}+t^{23}-t^{28}+t^{29}}{\left(1-t^{4}\right)^{2}\left(1-t^{6}\right)\left(1-t^{12}\right)}
\end{aligned}
$$




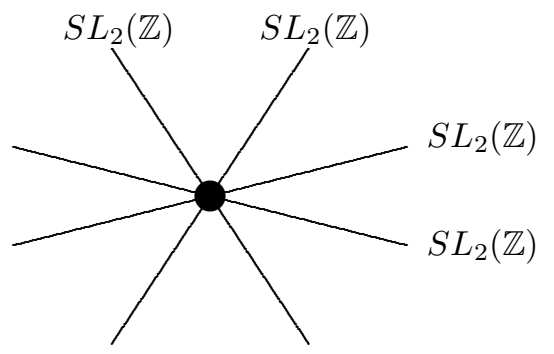

Figure 2. The cusp structure of structure of $K(N)$ for squarefree $N$.

$$
=t^{7}+t^{8}+t^{9}+2 t^{10}+3 t^{11}+4 t^{12}+3 t^{13}+5 t^{14}+6 t^{15}+10 t^{16}+\cdots .
$$

Proof. Satake's Theorem gives us:

$$
\operatorname{dim} M_{k}(K(4))-\operatorname{dim} S_{k}(K(4))=2+2 \operatorname{dim} S_{k}\left(\mathrm{SL}_{2}(\mathbb{Z})\right)+\operatorname{dim} S_{k}\left(\Gamma_{0}(2)\right)
$$

for even weights $k \geq 5$. The modular forms of odd weight are necessarily cusp forms. We use the known generating functions $\sum_{k \in \mathbb{Z}} \operatorname{dim} S_{k}\left(\mathrm{SL}_{2}(\mathbb{Z})\right) t^{k}=$ $t^{12} /\left(\left(1-t^{4}\right)\left(1-t^{6}\right)\right)$ and $\sum_{k \in \mathbb{Z}} \operatorname{dim} S_{k}\left(\Gamma_{0}(2)\right) t^{k}=t^{8} /\left(\left(1-t^{2}\right)\left(1-t^{4}\right)\right)$, along with the known generating function for $\sum_{k=0}^{\infty} \operatorname{dim} M_{k}(K(4)) t^{k}$, to obtain our conclusion for weights $k \geq 5$. For weights $k \leq 4$, only the weight four space is nontrivial. There cannot be a nontrivial cusp form in the two dimensional space of weight 4 because the cusp forms of weight 8 are one dimensional.

For squarefree $N$, the 1-cusps are spheres and all cross at a unique 0cusp as in Figure 2. By Satake's Theorem for even weights $k>4$ we have $\operatorname{dim} M_{k}(K(N))-\operatorname{dim} S_{k}(K(N))=1+\tau(N) \operatorname{dim} S_{k}\left(\mathrm{SL}_{2}(\mathbb{Z})\right)$. This cusp structure and our choice of global representatives will be made use of in [11], where Ibukiyama and Kitayama compute the dimensions $\operatorname{dim} S_{k}(K(N))$ for squarefree $N$ and $k \geq 4$. Previously, the dimensions $\operatorname{dim} S_{k}(K(N))$ were known for prime levels $N$ and $k \geq 3$ by the work of Ibukiyama (see [9, 10]). For prime levels, the structure of the cusps and their configuration is known for all parahoric subgroups of general degree (see Propositions 3.6 and 5.2 in Ibukiyama's article [8]). By piecing together the situation for prime levels, one may obtain a proof that paramodular groups of squarefree level have exactly one zero-cusp in arbitrary degree. We thank R. Schulze-Pillot for this observation.

In order to give context to the presentation of the cusp structure of the paramodular groups in degree two, let's review the situation in degree one for $\Gamma_{0}(\ell)$ and $\Gamma_{1}(\ell)$, compare [4]. We set

$$
P_{1,0}(\mathbb{Q})=\left\{\left(\begin{array}{ll}
* & * \\
0 & *
\end{array}\right): * \in \mathbb{Q}\right\} \cap S L_{2}(\mathbb{Q}) .
$$

In order to compactify $X_{0}(\ell)=\Gamma_{0}(\ell) \backslash \mathcal{H}_{1}$, we add a finite number of 0 -cusps $\Gamma_{0}(\ell) \backslash \mathbb{P}^{1}(\mathbb{Q})$. These 0 -cusps correspond to the double coset decomposition of 
$\Gamma_{0}(\ell) \backslash \mathrm{SL}_{2}(\mathbb{Z}) / P_{1,0}(\mathbb{Z})$, which is parameterized by the set

$$
(q, u) \in \coprod_{q \in \mathbb{N}: q \mid \ell}(\mathbb{Z} / \operatorname{gcd}(q, \ell / q) \mathbb{Z})^{\times}
$$

as follows. For each $q \mid \ell$, we select an $\hat{u} \in \mathbb{Z}$ prime to $\ell$ that gives the class of $u$ in $(\mathbb{Z} / \operatorname{gcd}(q, \ell / q) \mathbb{Z})^{\times}$and complete in any manner to a matrix $\left(\begin{array}{c}\hat{u} \\ q *\end{array}\right) \in \operatorname{SL}_{2}(\mathbb{Z})$. The corresponding disjoint double coset decomposition is independent of these choices:

$$
\mathrm{SL}_{2}(\mathbb{Z})=\bigcup_{q, u} \Gamma_{0}(\ell)\left(\begin{array}{ll}
\hat{u} & * \\
q & *
\end{array}\right) P_{1,0}(\mathbb{Z}) .
$$

For $X_{1}(\ell)=\Gamma_{1}(\ell) \backslash \mathcal{H}_{1}$, the cusps are parameterized by

$$
(q, u, d) \in \coprod_{q \in \mathbb{N}: q \mid \ell}(\mathbb{Z} / \operatorname{gcd}(q, \ell / q) \mathbb{Z})^{\times} \times(\mathbb{Z} / \ell \mathbb{Z})^{\times}
$$

and the disjoint double coset decomposition is

$$
\mathrm{SL}_{2}(\mathbb{Z})=\coprod_{q, u, d} \Gamma_{1}(\ell)\left(\begin{array}{ll}
* & * \\
* & \hat{d}
\end{array}\right)\left(\begin{array}{ll}
\hat{u} & * \\
q & *
\end{array}\right) P_{1,0}(\mathbb{Z}) .
$$

Here, $\hat{u}$ is as before and the integer $\hat{d}$ is prime to $\ell$ and gives the class of $d$ in $(\mathbb{Z} / \ell \mathbb{Z})^{\times}$. The matrix $\left(\begin{array}{c}* \\ * \\ *\end{array}\right) \in \Gamma_{0}(\ell)$ is completed from $\hat{d}$ in any manner.

For the paramodular group $K(N)$, the 1-cusps $K(N) \backslash \mathcal{H}_{2,1}^{*}$ correspond to the double cosets $K(N) \backslash \operatorname{Sp}_{2}(\mathbb{Q}) / P_{2,1}(\mathbb{Q})$, where

$$
P_{2,1}(\mathbb{Q})=\left\{\left(\begin{array}{llll}
* & 0 & * & * \\
* & * & * & * \\
* & 0 & * & * \\
0 & 0 & 0 & *
\end{array}\right): * \in \mathbb{Q}\right\} \cap S p_{2}(\mathbb{Q}) .
$$

As Reefschläger has shown, the number of 1-cusps is $\tau(N)$, the number of positive divisors of $N$. This number is perhaps more interesting than any particular choice of representatives for the double cosets in $K(N) \backslash \operatorname{Sp}_{2}(\mathbb{Q}) / P_{2,1}(\mathbb{Q})$ because, once the number of 1-cusps is known, any full number of inequivalent representatives must give a complete set of double coset representatives. For example, we have the following theorem.

Theorem 1.2 (Reefschläger). Let $N \in \mathbb{N}$. We have a disjoint union

$$
\mathrm{Sp}_{2}(\mathbb{Q})=\bigcup_{m \in \mathbb{N}: m \mid N} K(N) \mathcal{C}_{1}(m) P_{2,1}(\mathbb{Q}) \text {, with } \mathcal{C}_{1}(m)=\left(\begin{array}{cccc}
1 & m & 0 & 0 \\
0 & 1 & 0 & 0 \\
0 & 0 & 1 & 0 \\
0 & 0 & -m & 1
\end{array}\right)
$$

The number of 1-cusps is $\tau(N)$.

These coset representatives do have the nice property that for any integer $m$, $\mathcal{C}_{1}(m)$ and $\mathcal{C}_{1}(\operatorname{gcd}(m, N))$ represent the same double coset. In Theorem 4.3 , we complete the study of the 1-cusps by showing that the boundary component corresponding to a double coset as above is isomorphic to the compactification 
of $\tilde{\Gamma}_{1}(\ell) \backslash \mathcal{H}_{1}$, where the group is $\tilde{\Gamma}_{1}(\ell)=\left\{\sigma \in \mathrm{SL}_{2}(\mathbb{Z}): \sigma \equiv \pm\left(\begin{array}{ll}1 & * \\ 0 & 1\end{array}\right) \bmod \ell\right\}$ and $\ell=\operatorname{gcd}(m, N / m)$.

For the paramodular group $K(N)$, the 0 -cusps $K(N) \backslash \mathcal{H}_{2,0}^{*}$ correspond to the double cosets $K(N) \backslash \mathrm{Sp}_{2}(\mathbb{Q}) / P_{2,0}(\mathbb{Q})$, where

$$
P_{2,0}(\mathbb{Q})=\left\{\left(\begin{array}{llll}
* & * & * & * \\
* & * & * & * \\
0 & 0 & * & * \\
0 & 0 & * & *
\end{array}\right): * \in \mathbb{Q}\right\} \cap S p_{2}(\mathbb{Q}) .
$$

Theorem 1.3. Let $N, f, N_{0} \in \mathbb{N}$ with $N=f^{2} N_{0}$ and $N_{0}$ squarefree. We have a disjoint double coset decomposition

$$
\begin{aligned}
S p_{2}(\mathbb{Q}) & =\bigcup_{c, b} K(N) \mathcal{C}_{0}(\hat{b} c) P_{2,0}(\mathbb{Q}), \text { with } \mathcal{C}_{0}(x)=\left(\begin{array}{llll}
1 & 0 & 0 & 0 \\
0 & 1 & 0 & 0 \\
0 & x & 1 & 0 \\
x & 0 & 0 & 1
\end{array}\right), \\
(c, b) & \in \coprod_{c \in \mathbb{N}: c \mid f}(\mathbb{Z} / c \mathbb{Z})^{\times} /\{ \pm 1\},
\end{aligned}
$$

where $\hat{b}$ is prime to $N$ and gives the class of $b$ in $(\mathbb{Z} / c \mathbb{Z})^{\times} /\{ \pm 1\}$. The number of 0 -cusps is $1+\llcorner f / 2\lrcorner$.

These coset representatives have the following nice properties: For $m \mid N$ and $M$ prime to $N, K(N) \mathcal{C}_{0}(M m) P_{2,0}(\mathbb{Q})=K(N) \mathcal{C}_{0}(M \ell) P_{2,0}(\mathbb{Q})$ where $\ell=$ $\operatorname{gcd}(m, N / m)$. For $\ell_{i} \in \mathbb{N}$ with $\ell_{i}^{2} \mid N$ and integers $M_{i}$ prime to $N$, Proposition 5.3 proves that

$$
\begin{gathered}
K(N) \mathcal{C}_{0}\left(M_{1} \ell_{1}\right) P_{2,0}(\mathbb{Q})=K(N) \mathcal{C}_{0}\left(M_{2} \ell_{2}\right) P_{2,0}(\mathbb{Q}) \\
\text { if and only if } \ell_{1}=\ell_{2} \text { and } M_{1} \equiv \pm M_{2} \bmod \ell_{1} .
\end{gathered}
$$

The incidence relations among the boundary components of the Satake compactification follow from these properties. For a divisor $m \mid N$, set $\ell=$ $\operatorname{gcd}(m, N / m)$. The boundary curve of the Satake compactification corresponding to a divisor $m \mid N$ is isomorphic to the modular curve $\tilde{\Gamma}_{1}(\ell) \backslash \mathcal{H}_{1}$, whose 0 -cusps are of the form $\tilde{\Gamma}_{1}(\ell)\left(\begin{array}{l}* * \\ * \hat{d}\end{array}\right)\left(\begin{array}{l}\hat{u} * \\ q *\end{array}\right) P_{1,0}(\mathbb{Z})$ with $q$ and $u$ as in the case of $\Gamma_{0}(\ell)$, and $\hat{d}$, which we may pick prime to $N$, running over the classes of $(\mathbb{Z} / \ell \mathbb{Z})^{\times} /\{ \pm 1\}$. In the Satake compactification, $\mathcal{S}\left(K(N) \backslash \mathcal{H}_{2}\right)$, the 0 -cusps are identified according to:

$$
\tilde{\Gamma}_{1}(\ell)\left(\begin{array}{cc}
* & * \\
* & \hat{d}
\end{array}\right)\left(\begin{array}{ll}
\hat{u} & * \\
q & *
\end{array}\right) P_{1,0}(\mathbb{Z}) \mapsto K(N) \mathcal{C}_{0}\left(\hat{d} \frac{\ell}{q}\right) P_{2,0}(\mathbb{Q}) .
$$

This completely describes the crossing of the boundary curves at the 0-cusps, compare Theorem 6.2. On the other hand, given a 0-cusp, we can count the number of times each boundary curve crosses this 0 -cusp. This final result is given as Theorem 1.4. For $n \in \mathbb{N}$, define

$$
\varphi^{\prime}(n)=\left|(\mathbb{Z} / n \mathbb{Z})^{\times} /( \pm 1)\right|= \begin{cases}1 & \text { if } n \leq 2 \\ \varphi(n) / 2 & \text { if } n>2 .\end{cases}
$$


The reader may enjoy verifying that $\sum_{c \mid f} \phi^{\prime}(c)=1+\llcorner f / 2\lrcorner$, so that the number of zero-dimensional cusps of $\mathcal{S}\left(K(N) \backslash \mathcal{H}_{2}\right)$ is indeed $1+\llcorner f / 2\lrcorner$, with $N=f^{2} N_{0}$ for $f, N_{0} \in \mathbb{N}$ and $N_{0}$ squarefree.

Theorem 1.4. Let $N \in \mathbb{N}$. Fix $m \mid N$ and the one-dimensional cusp corresponding to $K(N) \mathcal{C}_{1}(m) P_{2,1}(\mathbb{Q})$. Call $\ell=\operatorname{gcd}(m, N / m)$. Its inequivalent zero-dimensional cusps all correspond to $K(N) \mathcal{C}_{0}(b c) P_{2,0}(\mathbb{Q})$ for $c \mid \ell$ and $\operatorname{gcd}(b, N)=1$ where $b$ runs over distinct elements of the group $(\mathbb{Z} / \ell \mathbb{Z})^{\times} /( \pm 1)$. Each zero-dimensional cusp is crossed by the curve the following number of times,

which is

$$
\frac{\varphi^{\prime}(\operatorname{lcm}(c, \ell / c)) \varphi(\operatorname{gcd}(c, \ell / c))}{\varphi^{\prime}(c)}
$$

$$
\begin{cases}\frac{1}{2} \varphi(\ell / c) & \text { if } c \leq 2 \text { and } \ell>2 c, \\ \frac{\varphi(\operatorname{lcm}(c, \ell / c)) \varphi(\operatorname{gcd}(c, \ell / c))}{\varphi(c)} & \text { otherwise. }\end{cases}
$$

We thank T. Ibukiyama for encouraging the second author to write this article at the RIMS Conference, Automorphic forms, trace formulas and zeta functions in Kyoto, Japan during January of 2011. We thank R. Schulze-Pillot for obtaining Reefschläger's thesis [14] for us.

\section{Notation}

We set $J=\left(\begin{array}{cccc}0 & 0 & 1 & 0 \\ 0 & 0 & 0 & 1 \\ -1 & 0 & 0 & 0 \\ 0 & -1 & 0 & 0\end{array}\right)$ and $J_{N}=\left(\begin{array}{cccc}0 & 0 & 1 & 0 \\ 0 & 0 & 0 & 1 / N \\ -1 & 0 & 0 & 0 \\ 0 & -N & 0 & 0\end{array}\right)$. We largely follow Freitag's textbook [5]. For example, the symplectic group over a ring $R$ is $\operatorname{Sp}_{n}(R)=\left\{\gamma \in \mathrm{GL}_{2 n}(R): \gamma^{\prime} J \gamma=J\right\}$ and for a subgroup $G \subseteq \operatorname{Sp}_{n}(\mathbb{R})$, we call $G^{\mathrm{pr}}=G \cap \mathbb{R}_{+} M_{2 n \times 2 n}(\mathbb{Q})$ the projective rational elements of $G$. Useful types of symplectic matrices are $u(A)=\left(\begin{array}{cc}A & 0 \\ 0 & A^{*}\end{array}\right)$ for $A \in G L_{2}(\mathbb{R})$, and $t(B)=\left(\begin{array}{cc}I & B \\ 0 & I\end{array}\right)$ for $B \in M_{2 \times 2}^{\text {sym }}(\mathbb{R})$. Besides the standard congruence subgroups we use

$$
\begin{aligned}
\tilde{\Gamma}_{1}(\ell) & =\left\{A \in \mathrm{SL}_{2}(\mathbb{Z}): A \equiv\left(\begin{array}{ll}
1 & * \\
0 & 1
\end{array}\right) \bmod \ell \text { or } A \equiv\left(\begin{array}{cc}
-1 & * \\
0 & -1
\end{array}\right) \bmod \ell\right\}, \\
G(\ell, \delta) & =\left(\begin{array}{ll}
1 & 0 \\
0 & \delta
\end{array}\right) \tilde{\Gamma}_{1}(\ell)\left(\begin{array}{ll}
1 & 0 \\
0 & \delta
\end{array}\right)
\end{aligned}
$$

for $\ell, \delta \in \mathbb{N}$. For a set $U$ and subgroups $B_{q} \subseteq U$ indexed by $q \in A$, we use a disjoint union in the form $\coprod_{q \in A} B_{q}=\cup_{q \in A} \cup_{x \in B_{q}}(q, x) \subseteq A \times U$. For two square matrices $A$ and $B$, we set $A \perp B=\left(\begin{array}{cc}A & 0 \\ 0 & B\end{array}\right)$.

\section{Satake compactification}

To review the Satake compactification, define the Grassmannian of rank $n$ subspaces of $\mathbb{C}^{2 n}, \operatorname{Gr}_{\mathbb{C}}(2 n, n)=M_{2 n \times n}^{\text {rank } n}(\mathbb{C}) / \mathrm{GL}_{n}(\mathbb{C})$, as well as $\operatorname{Gr}_{\mathbb{C}}^{\text {iso }}(2 n, n)=$ $\left\{\left[\begin{array}{c}M \\ N\end{array}\right] \in \operatorname{Gr}_{\mathbb{C}}(2 n, n): J\left[\begin{array}{c}M \\ N\end{array}\right]=0\right\}$, the Grassmannian of isotropic rank $n$ subspaces. The symplectic group $\operatorname{Sp}_{n}(\mathbb{C})$ has a natural left action on $\operatorname{Gr}_{\mathbb{C}}^{\text {iso }}(2 n, n)$. Let $V_{n}(\mathbb{C})=M_{n \times n}^{\mathrm{sym}}(\mathbb{C})$ be the vector space of $n$-by- $n$ symmetric matrices viewed as a subset of the isotropic Grassmannian via the identification $V_{n}(\mathbb{C}) \ni W \mapsto$ 
$\left[\begin{array}{c}W \\ I\end{array}\right] \in \operatorname{Gr}_{\mathbb{C}}^{\text {iso }}(2 n, n)$. For $m=0,1,2, \ldots, n$, we define analytic maps (by convention, $\left.\mathcal{H}_{0}=\{\infty\}\right)$

$$
\begin{aligned}
j_{n, m}: \mathcal{H}_{m} & \rightarrow \operatorname{Gr}_{\mathbb{C}}^{\mathrm{iso}}(2 n, n) \\
Z & \mapsto\left[\begin{array}{cc}
I \\
\left(\begin{array}{cc}
I-1 & 0 \\
0 & 0
\end{array}\right)
\end{array}\right] .
\end{aligned}
$$

Note that $j_{n, n}: \mathcal{H}_{n} \rightarrow \operatorname{Gr}_{\mathbb{C}}^{\text {iso }}(2 n, n)$ coincides with the embedding of $\mathcal{H}_{n} \subseteq$ $V_{n}(\mathbb{C}) \subseteq \operatorname{Gr}_{\mathbb{C}}^{\text {iso }}(2 n, n)$. These $j_{n, m}$ are introduced because, setting $Z_{n}=Z_{m} \perp$ $i \lambda I_{n-m} \in \mathcal{H}_{n}$ for $Z_{m} \in \mathcal{H}_{m}$ and letting $\lambda \rightarrow+\infty$, it is clear that $j_{n, m}\left(\mathcal{H}_{m}\right)$ is in the closure of $j_{n, n}\left(\mathcal{H}_{n}\right)$. Among the $j_{n, m}\left(\mathcal{H}_{m}\right)$ for $0 \leq m \leq n$, only the image of $\mathcal{H}_{n}$ is stable under $\operatorname{Sp}_{n}(\mathbb{R})^{\mathrm{pr}}$ and so we define $\mathcal{H}_{n, m}^{*}=\overline{\operatorname{Sp}}_{n}(\mathbb{R})^{\mathrm{pr}} j_{n, m}\left(\mathcal{H}_{m}\right)$. Consequently, the disjoint union

$$
\mathcal{S}=\mathcal{H}_{n, n}^{*} \cup \mathcal{H}_{n, n-1}^{*} \cup \cdots \cup \mathcal{H}_{n, 1}^{*} \cup \mathcal{H}_{n, 0}^{*} \subseteq \operatorname{Gr}_{\mathbb{C}}^{\text {iso }}(2 n, n)
$$

is stable under the action of $\operatorname{Sp}_{n}(\mathbb{R})^{\mathrm{pr}}$. Let $\Gamma \subseteq \mathrm{Sp}_{n}(\mathbb{R})^{\mathrm{pr}}$ be a subgroup commensurable with $\operatorname{Sp}_{n}(\mathbb{Z})$. We define $\mathcal{S}\left(\Gamma \backslash \mathcal{H}_{n}\right)=\Gamma \backslash \mathcal{S}$ to be the Satake compactification of $\Gamma \backslash \mathcal{H}_{n}$. This is well-defined because elements of $\Gamma$ that act trivially on $\mathcal{H}_{n}$ also act trivially on $\mathcal{S}$. Satake defined an intrinsic topology on $\mathcal{S}$ and proved that $\Gamma \backslash \mathcal{S}$ is indeed compact in the quotient topology (see $[16,17]$ ). Furthermore, this quotient topology on $\Gamma \backslash \mathcal{S}$ agrees with the topology induced by $\Gamma \backslash \operatorname{Gr}_{\mathbb{C}}^{\text {iso }}(2 n, n)$ and $\mathcal{S}\left(\Gamma \backslash \mathcal{H}_{n}\right)$ is the closure of $\Gamma \backslash \mathcal{H}_{n, n}^{*}$ inside $\Gamma \backslash \operatorname{Gr}_{\mathbb{C}}^{\text {iso }}(2 n, n)$. One can check that the stabilizer inside $\operatorname{Sp}_{n}(\mathbb{R})^{\text {pr }}$ of $j_{n, m}\left(\mathcal{H}_{m}\right)$ is $P_{n, m}(\mathbb{R})^{\text {pr }}$ where

$$
P_{n, m}(\mathbb{F})=\left\{\left(\begin{array}{cccc}
A_{11} & 0 & B_{11} & B_{12} \\
A_{21} & A_{22} & B_{21} & B_{22} \\
C_{11} & 0 & D_{11} & D_{12} \\
0 & 0 & 0 & D_{22}
\end{array}\right) \in \operatorname{Sp}_{n}(\mathbb{F})\right\}
$$

We have a homomorphism $\omega_{n, m}: P_{n, m}(\mathbb{R})^{\mathrm{pr}} \rightarrow \mathrm{Sp}_{m}(\mathbb{R})^{\mathrm{pr}}$, defined by sending $\sigma \mapsto\left(\begin{array}{ll}A_{11} & B_{11} \\ C_{11} & D_{11}\end{array}\right)$, that satisfies $\sigma j_{n, m}(Z)=j_{n, m}\left(\omega_{n, m}(\sigma) Z\right)$ for all $Z \in \mathcal{H}_{m}$ and $\sigma \in P_{n, m}(\mathbb{R})^{\text {pr }}$. We call the kernel $H_{n, m}$ of $\omega_{n, m}$ a Heisenberg group. As a set we have $\mathcal{H}_{n, m}^{*}=\cup_{\gamma} \Gamma \gamma j_{n, m}\left(\mathcal{H}_{m}\right)$ where $\gamma$ runs over representatives for $\Gamma \backslash \operatorname{Sp}_{n}(\mathbb{R})^{\mathrm{pr}} / \operatorname{Stab}\left(j_{n, m}\left(\mathcal{H}_{m}\right)\right)$, say $\operatorname{Sp}_{n}(\mathbb{R})^{\mathrm{pr}}=\cup_{\gamma} \Gamma \gamma P_{n, m}(\mathbb{R})^{\mathrm{pr}}$. In this way, the components of $\Gamma \backslash \mathcal{H}_{n, m}^{*}$ correspond to a double coset decomposition and it is these double cosets that we usually work with. For each piece of this double coset decomposition, setting $\Gamma_{\gamma}=\omega_{n, m}\left(\gamma^{-1} \Gamma \gamma \cap P_{n, m}(\mathbb{R})^{\text {pr }}\right)$, we have the isomorphism

$$
\Psi_{n, m, \gamma}: \Gamma_{\gamma} \backslash \mathcal{H}_{m} \cong \Gamma \backslash \Gamma \gamma j_{n, m}\left(\mathcal{H}_{m}\right) \subseteq \mathcal{S}\left(\Gamma \backslash \mathcal{H}_{n}\right),
$$

given by sending $\Gamma_{\gamma} Z \mapsto \Gamma \gamma j_{n, m}(Z)$. This gives the boundary components the structure of modular varieties and these varieties, or often their closures, are referred to as the $m$-cusps of $\mathcal{S}\left(\Gamma \backslash \mathcal{H}_{n}\right)$.

Now $\Gamma_{\gamma} \subseteq \operatorname{Sp}_{m}(\mathbb{R})^{\text {pr }}$ is commensurable with $\operatorname{Sp}_{m}(\mathbb{Z})$ and so has its own Satake compactification $\mathcal{S}\left(\Gamma_{\gamma} \backslash \mathcal{H}_{m}\right)$. In order to extend $\Psi_{n, m, \gamma}$ to $\mathcal{S}\left(\Gamma_{\gamma} \backslash \mathcal{H}_{m}\right)$, 
we need the homomorphism $i_{n, m}: \mathrm{Sp}_{m}(\mathbb{R}) \rightarrow P_{n, m}(\mathbb{R})$ defined by

$$
\left(\begin{array}{cc}
A & B \\
C & D
\end{array}\right) \mapsto\left(\begin{array}{cccc}
A & 0 & B & 0 \\
0 & I_{n-m} & 0 & 0 \\
C & 0 & D & 0 \\
0 & 0 & 0 & I_{n-m}
\end{array}\right) .
$$

The map $\Psi_{n, m, \gamma}$ extends to a continuous map $\Psi_{n, m, \gamma}: \mathcal{S}\left(\Gamma_{\gamma} \backslash \mathcal{H}_{m}\right) \rightarrow \mathcal{S}\left(\Gamma \backslash \mathcal{H}_{n}\right)$ and the image of $\mathcal{S}\left(\Gamma_{\gamma} \backslash \mathcal{H}_{m}\right)$ is the closure of $\Gamma \backslash \Gamma \gamma j_{n, m}\left(\mathcal{H}_{m}\right)$ inside $\mathcal{S}\left(\Gamma \backslash \mathcal{H}_{n}\right)$. The extension $\Psi_{n, m, \gamma}: \Gamma_{\gamma} \backslash \mathcal{H}_{m, \ell}^{*} \rightarrow \Gamma \backslash \mathcal{H}_{n, \ell}^{*}$ is defined by sending $\Gamma_{\gamma} \rho j_{m, \ell}(Z) \mapsto$ $\Gamma \gamma i_{n, m}(\rho) j_{n, \ell}(Z)$ for $Z \in \mathcal{H}_{\ell}$ and $\rho \in \operatorname{Sp}_{m}(\mathbb{R})^{\text {pr }}$. One can show this map is welldefined by using the identities: $j_{n, \ell}\left(\omega_{m, \ell}(\tau) Z\right)=i_{n, m}(\tau) j_{n, \ell}(Z)$ for all $Z \in \mathcal{H}_{\ell}$ and all $\tau \in P_{m, \ell}(\mathbb{R})^{\mathrm{pr}}$; and $i_{n, m}\left(\omega_{n, m}(\sigma)\right) \in \sigma H_{n, m}$ for all $\sigma \in P_{n, m}(\mathbb{R})^{\mathrm{pr}}$.

Thus we may consider cusps of cusps. We may have an $m$-cusp with an $\ell$-cusp of its own that we wish to identify as a boundary component of $\mathcal{S}\left(\Gamma \backslash \mathcal{H}_{n}\right)$. For $n>m>\ell$ and $\gamma \in \operatorname{Sp}_{n}(\mathbb{R})^{\text {pr }}$, consider an $\ell$-cusp of $\mathcal{S}\left(\Gamma_{\gamma} \backslash \mathcal{H}_{m}\right)$ corresponding to a double coset $\Gamma_{\gamma} \rho P_{m, \ell}(\mathbb{R})^{\text {pr }}$ with $\rho \in \operatorname{Sp}_{m}(\mathbb{R})^{\text {pr }}$. The $\ell$-cusp as a boundary component of $\mathcal{S}\left(\Gamma \backslash \mathcal{H}_{n}\right)$ corresponds to the double coset $\Gamma \gamma i_{n, m}(\rho) P_{n, \ell}(\mathbb{R})^{\mathrm{pr}}$. If this is the same double coset for inequivalent representatives $\gamma$, then it means that two $m$-cusps intersect at this $\ell$-cusp; for equivalent $\gamma$ but inequivalent $\rho$, it means that the $m$-cusp has self intersection.

An immediate virtue of the Satake compactification is the existence of a global $\Phi$-map. We consider only the special case where $m=n-1, \ell=n-$ 2 and $\Gamma \subseteq \operatorname{Sp}_{n}(\mathbb{Q})$, appropriate for degree two paramodular groups. The usual map $\Phi: M_{k}(\Gamma) \rightarrow M_{k}\left(\omega_{n, n-1}\left(\Gamma \cap P_{n, n-1}(\mathbb{Q})\right)\right)$ is defined by $(\Phi f)(Z)=$ $\lim _{\lambda \rightarrow+\infty} f(Z \perp i \lambda)$ for $Z \in \mathcal{H}_{n-1}$. This maps satisfies

$$
\Phi^{(n-r)}(f \mid \delta)=\left(\Phi^{(n-r)}(f)\right) \mid \omega_{n, r}(\delta)
$$

for $\delta \in P_{n, r}(\mathbb{Q})$ and integers $r=0,1, \ldots, n-1$. We now fix representatives $\gamma_{i}$ for a disjoint double coset decomposition $\operatorname{Sp}_{n}(\mathbb{Q})=\cup_{i=1}^{p} \Gamma \gamma_{i} P_{n, n-1}(\mathbb{Q})$ and set $\Gamma_{i}=\omega_{n, n-1}\left(\gamma_{i}^{-1} \Gamma \gamma_{i} \cap P_{n, n-1}(\mathbb{Q})\right)$. Define $\tilde{\Phi}: M_{k}(\Gamma) \rightarrow \oplus_{i=1}^{p} M_{k}\left(\Gamma_{i}\right)$ by $f \mapsto \prod_{i=1}^{p} \Phi\left(f \mid \gamma_{i}\right)$. Any element $\prod_{i=1}^{p} f_{i} \in \operatorname{Im} \tilde{\Phi}$ satisfies the following condition:

$$
\begin{array}{r}
\forall 1 \leq i, j \leq p, \forall h_{1}, h_{2} \in \operatorname{Sp}_{n-1}(\mathbb{Q}), \text { we have } \Phi\left(f_{i} \mid h_{1}\right)=\Phi\left(f_{j} \mid h_{2}\right) \\
\text { whenever } \Gamma \gamma_{i} i_{n, n-1}\left(h_{1}\right) P_{n, n-2}(\mathbb{Q})=\Gamma \gamma_{j} i_{n, n-1}\left(h_{2}\right) P_{n, n-2}(\mathbb{Q}) .
\end{array}
$$

This condition amounts to saying that the $f_{i}$ agree where the cusps cross. In [18], Satake proved that for even weights $k>4$ the condition (1) characterizes the image of the global $\tilde{\Phi}$ map. The restriction on the weight in his proof arises from the need to have convergent Poincare series; for some relaxation to $k=4$ in degree two see [1]. 


\section{The one dimensional cusps}

We review Reefschläger's result that the 1-cusps of a paramodular group of level $N$ correspond to divisors of $N$. It is possible to do this briefly because the proof has been streamlined by Gritsenko, who introduced a useful invariant. The paramodular group $K(N)$ stabilizes the lattice $L_{N}=\operatorname{diag}(1,1,1, N) \mathbb{Z}^{4}$ and the transposed group $K(N)^{\prime}$ stabilizes the dual lattice $L_{N}^{*}=\operatorname{diag}(1,1,1,1 / N) \mathbb{Z}^{4}$. For $X \in \mathbb{Q}^{4}$, the fractional ideals $X^{\prime} J L_{N}$ and $X^{\prime} L_{N}^{*}$ are invariants of the orbit $K(N) X$ because $(\sigma X)^{\prime} J L_{N}=X^{\prime} \sigma^{\prime} J L_{N}=X^{\prime} J \sigma^{-1} L_{N}=X^{\prime} J L_{N}$ and $(\sigma X)^{\prime} L_{N}^{*}=X^{\prime} \sigma^{\prime} L_{N}^{*}=X^{\prime} L_{N}^{*}$. These fractional ideals both scale by $\alpha$ when we replace $X$ by $\alpha X$ for $\alpha \in \mathbb{Q}^{*}$. In order to remove this scaling factor, define $1-\operatorname{inv}(\mathrm{X})=\left(X^{\prime} J L_{N}\right)\left(X^{\prime} L_{N}^{*}\right)^{-1}$; this fractional ideal is an invariant of the orbit $K(N)[X]$ for $[X] \in \mathbb{P}^{3}(\mathbb{Q})$ and is in fact an integral ideal containing $N \mathbb{Z}$ because $J L_{n} \supseteq N L_{N}^{*}$. The following algorithmic result is due to Gritsenko (see $[6])$.

Proposition 4.1 (Gritsenko). Let $N \in \mathbb{N}$ and $[X] \in \mathbb{P}^{3}(\mathbb{Q})$. We have

$$
\left.K(N)[X]=K(N)\left[\begin{array}{llll}
(d & 1 & 0 & 0
\end{array}\right)^{\prime}\right]
$$

for $1-\operatorname{inv}(X)=d \mathbb{Z} \supseteq N \mathbb{Z}$.

This result classifies $K(N) \backslash S p_{2}(\mathbb{Q}) / P_{2,1}(\mathbb{Q})$ as well. Denote the second column of $\sigma \in \operatorname{Sp}_{2}(\mathbb{Q})$ by $\sigma_{2}$; then defining $1-\operatorname{inv}(\sigma)=1-\operatorname{inv}\left(\sigma_{2}\right)$, this is an invariant of the double coset $K(N) \sigma P_{2,1}(\mathbb{Q})$. Proposition 4.1 implies that this invariant separates the double cosets as well.

Proposition 4.2. Let $N \in \mathbb{N}, \sigma \in \operatorname{Sp}_{2}(\mathbb{Q})$. We have $K(N) \sigma P_{2,1}(\mathbb{Q})=$ $K(N) u\left(\begin{array}{ll}1 & d \\ 0 & 1\end{array}\right) P_{2,1}(\mathbb{Q})$ for $1-\operatorname{inv}(\sigma)=d \mathbb{Z} \supseteq N \mathbb{Z}$. Furthermore, for $d \mid N$, we have 1 -inv $\left(u\left(\begin{array}{ll}1 & d \\ 0 & 1\end{array}\right)\right)=d \mathbb{Z}$.

Proof. Take $\sigma \in \mathrm{Sp}_{2}(\mathbb{Q})$. By Proposition 4.1, there exists a $\gamma \in K(N)$ such that $(\gamma \sigma)_{2}=\alpha\left(\begin{array}{llll}d & 1 & 0 & 0\end{array}\right)^{\prime}$ for $d \mathbb{Z}=1-\operatorname{inv}\left(\sigma_{2}\right)=1-\operatorname{inv}(\sigma)$ and $\alpha \in \mathbb{Q}^{*}$. Then $\left(u\left(\begin{array}{ll}1 & d \\ 0 & 1\end{array}\right)^{-1} \gamma \sigma\right)_{2}=\left(\begin{array}{llll}0 & \alpha & 0 & 0\end{array}\right)^{\prime}$ and so $u\left(\begin{array}{ll}1 & d \\ 0 & 1\end{array}\right)^{-1} \gamma \sigma \in P_{2,1}(\mathbb{Q})$, as easily follows from the symplectic conditions.

The disjoint double coset decomposition

$$
\operatorname{Sp}_{2}(\mathbb{Q})=\bigcup_{d \in \mathbb{N}: d \mid N} K(N) u\left(\begin{array}{ll}
1 & d \\
0 & 1
\end{array}\right) P_{2,1}(\mathbb{Q}),
$$

of Theorem 1.2 follows from Proposition 4.2. The use of the double coset invariant 1-inv has streamlined the exposition so that the essentially algorithmic nature of the double coset decomposition is confined to Proposition 4.1 and invoked only once. In the Appendix we present this algorithm explicitly enough to write a computer program to implement it. One should not expect every classification of double cosets to be reformulated so efficiently in terms of invariants. Even for $\Gamma_{0}(\ell)$ in degree one, one does not proceed wholly by invariants. The group $\Gamma_{0}(\ell)$ acts on the lattice $L_{\ell}=\mathbb{Z} \oplus \ell \mathbb{Z}$ and $\Gamma_{0}(\ell)^{\prime}$ acts 
on $L_{\ell}^{*}=\mathbb{Z} \oplus \frac{1}{\ell} \mathbb{Z}$, so that $X^{\prime} J L_{\ell}$ and $X^{\prime} L_{\ell}^{*}$ are ideals that are invariants of the orbit $\Gamma_{0}(\ell) X$ for $X \in \mathbb{Z}^{2}$. Applied to the first column, these invariants are enough to give the divisor $q \mid \ell$ of a normal form $\Gamma_{0}(\ell)\left(\begin{array}{cc}\hat{u} & * \\ q & *\end{array}\right) P_{1,0}(\mathbb{Z})$. The entry $\hat{u}$ is then varied by actions that fix $q$ and attempts to define a single complete invariant on $\Gamma_{0}(\ell) \backslash \mathrm{SL}_{2}(\mathbb{Z}) / P_{1,0}(\mathbb{Z})$ do little more than restate the classification problem. We now complete the classification of the 1-cusps by identifying the one dimensional boundary component corresponding to each positive divisor of $N$.

Theorem 4.3. Let $N, m \in \mathbb{N}$ with $m \mid N$ and set $\ell=\operatorname{gcd}(m, N / m)$. Let $\delta=\frac{1}{\ell} \frac{N}{m} \in \mathbb{N}$ so that $N=m \ell \delta$. Denote $\gamma=\mathcal{C}_{1}(m)$. Then

$$
\Gamma_{\gamma}=\omega_{2,1}\left(\gamma^{-1} K(N) \gamma \cap P_{2,1}(\mathbb{Q})\right)=\left(\begin{array}{ll}
1 & 0 \\
0 & \delta
\end{array}\right) \tilde{\Gamma}_{1}(\ell)\left(\begin{array}{ll}
1 & 0 \\
0 & \delta
\end{array}\right)^{-1} .
$$

Proof. Take any $\left(\begin{array}{ll}a & b \\ c & d\end{array}\right) \in \Gamma_{\gamma}$. There exists a preimage

$$
\left(\begin{array}{llll}
a & 0 & b & e \\
f & g & h & p \\
c & 0 & d & q \\
0 & 0 & 0 & r
\end{array}\right) \in \gamma^{-1} K(N) \gamma \cap P_{2,1}(\mathbb{Q}),
$$

where $a, b, c, d, e, f, g, h, p, q, r \in \mathbb{Q}$. Then the matrix

$$
\gamma\left(\begin{array}{llll}
a & 0 & b & e \\
f & g & h & p \\
c & 0 & d & q \\
0 & 0 & 0 & r
\end{array}\right) \gamma^{-1}=\left(\begin{array}{cccc}
a+f m & m(-a+g-f m) & b+e m+h m+p m^{2} & e+m p \\
f & g-f m & h+p m & p \\
c & -c m & d+q m & q \\
-c m & c m^{2} & m(-d+r-q m) & r-q m
\end{array}\right)
$$

is in $K(N)$. This implies that $f, c, q \in \mathbb{Z}$ and then $a, g, d, r \in \mathbb{Z}$ follow. From $e+m p, h+m p \in \mathbb{Z}$, and from

$$
b+e m+h m+m^{2} p=b-m^{2} p+m(e+m p+h+m p),
$$

we conclude that $b-m^{2} p \in \mathbb{Z}$. The paramodular divisibility condition $p \in \frac{1}{N} \mathbb{Z}$ then implies that $b \in \frac{\operatorname{gcd}\left(m^{2}, N\right)}{N} \mathbb{Z}=\frac{1}{\delta} \mathbb{Z}$. The paramodular divisibility condition $N \mid(-c m)$ implies $\frac{N}{m} \mid c$, or

$$
\ell \delta \mid c \text {. }
$$

We have $b c \in \ell \mathbb{Z}$ and, in particular, $b c \in \mathbb{Z}$. The determinant of the above 4 -by-4 matrix is $(a d-b c) g r$, which must be 1 . Then $a d-b c, g, r \in \mathbb{Z}$ forces $a d-b c, g, r= \pm 1$. Furthermore, the symplectic condition forces $a d-b c=1$ and $g=r= \pm 1$. Now, looking at the (1,2)-entry, we see that $\frac{N}{m} \mid(-a+g-f m)$. Since $\ell \mid m$ and $\ell \mid \frac{N}{m}$, then $\ell \mid(g-a)$. Similarly, looking at the (4,3)-entry, we deduce that $\ell \mid(d-r)$. Hence

$$
a \equiv d \equiv r \quad \bmod \ell,
$$

where $r=1$ or $r=-1$. Thus $\Gamma_{\gamma} \subseteq\left(\begin{array}{ll}1 & 0 \\ 0 & \delta\end{array}\right) \tilde{\Gamma}_{1}(\ell)\left(\begin{array}{ll}1 & 0 \\ 0 & \delta\end{array}\right)^{-1}$ because

$$
\left(\begin{array}{ll}
1 & 0 \\
0 & \delta
\end{array}\right)^{-1}\left(\begin{array}{ll}
a & b \\
c & d
\end{array}\right)\left(\begin{array}{ll}
1 & 0 \\
0 & \delta
\end{array}\right)=\left(\begin{array}{cc}
a & b \delta \\
c / \delta & d
\end{array}\right) \in \tilde{\Gamma}_{1}(\ell) .
$$

Conversely, take any $\left(\begin{array}{ll}a & b \\ c & d\end{array}\right) \in G(\ell, \delta)$. Then $a, c, d \in \mathbb{Z}$ and $b \in \frac{1}{\delta} \mathbb{Z}$ where $\ell \delta \mid c$ and $a \equiv d \equiv r \bmod \ell$ for some $r= \pm 1$. Let $b=b_{0} / \delta$ and $c=c_{0} \ell \delta$ where 
$b_{0}, c_{0} \in \mathbb{Z}$. Since $\operatorname{gcd}\left(\frac{m}{\ell}, \delta\right)=1$, there exist $k, z \in \mathbb{Z}$ such that $b_{0}=\frac{m}{\ell} k+\delta z$. Since $\ell \mid(r-d)$ and $\ell \mid(r-a)$, there exist $q_{0}, t_{0}, f, x \in \mathbb{Z}$ such that

$$
r-d=m q_{0}+\delta \ell t_{0} \text { and } r-a=m f+x \ell \delta .
$$

For $u, y \in \mathbb{Z}$ to be chosen later, let

$$
B=\left(\begin{array}{cccc}
r-\ell \delta x & N x & m u+z & u \\
f & r-f m & 0 & k / N \\
c_{0} \ell \delta & -c_{0} N & r-\ell \delta t_{0}+m \delta y & q_{0}+\delta y \\
-c_{0} N & c_{0} m N & N\left(t_{0}-\frac{m}{\ell} y\right) & r-m q_{0}-m \delta y
\end{array}\right)
$$

The conjugate of $B$ by $\gamma$ is:

$$
\gamma^{-1} B \gamma=\left(\begin{array}{cccc}
a & 0 & b & \frac{\ell \delta u-k}{\ell \delta} \\
f & r & \frac{-k}{\ell \delta} & k / N \\
c & 0 & d & q_{0}+\delta y \\
0 & 0 & 0 & r
\end{array}\right)
$$

Note $\omega_{1}\left(\gamma^{-1} B \gamma\right)=\left(\begin{array}{ll}a & b \\ c & d\end{array}\right)$, and this is independent of $u, y \in \mathbb{Z}$. If we can find $u, y \in \mathbb{Z}$ such that $\gamma^{-1} B \gamma \in S p_{2}(\mathbb{Q})$, then $B \in S p_{2}(\mathbb{Q})$ and so $B \in K(N)$ and thus $\omega_{1}\left(\gamma^{-1} B \gamma\right) \in \Gamma_{\gamma}$ and the proof would be complete. The matrix $\gamma^{-1} B \gamma$ being symplectic requires that

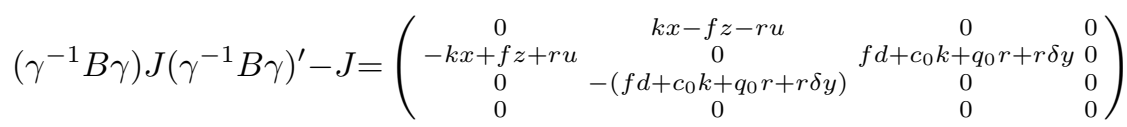

vanishes. To make $B$ symplectic, we need to make $k x-f z-r u=0$ and $f d+c_{0} k+q_{0} r+r \delta y=0$. Since $r= \pm 1$, we can set $u=r(k x-f z)$ to make $k x-f z-r u=0$. Next we claim $\delta \mid\left(f d+c_{0} k+q_{0} r\right)$ so that we can define $y=-\frac{r}{\delta}\left(f d+c_{0} k+q_{0} r\right) \in \mathbb{Z}$ to make $f d+c_{0} k+q_{0} r+r \delta y=0$. To see this, call $X=f d+c_{0} k+q_{0} r$. Then

$$
\begin{aligned}
m X & =m f d+m c_{0} k+m q_{0} r \\
& =(r-a-x \ell \delta) d+m c_{0} k+\left(r-d-\delta \ell t_{0}\right) r \\
& =1-a d+c_{0} k m-\ell \delta r t_{0}-d \ell \delta x \\
& =-b_{0} \ell c_{0}+c_{0} k m-\ell \delta r t_{0}-d \ell \delta x \\
& =-\ell \delta c_{0} z-\ell \delta r t_{0}-d \ell \delta x .
\end{aligned}
$$

Therefore $\ell \delta \mid m X$ and $\delta \mid \frac{m}{\ell} X$ follows. Since $\operatorname{gcd}\left(\delta, \frac{m}{\ell}\right)=1$, it follows that $\delta \mid X$ and this completes the proof.

The group $\tilde{\Gamma}_{1}(\ell)$ has come up in a natural manner and we will need to have good control over its cusps.

Lemma 4.4. We have the disjoint double coset decomposition

$$
\mathrm{SL}_{2}(\mathbb{Z})=\bigcup_{q, u, d} \tilde{\Gamma}_{1}(\ell)\left(\begin{array}{ll}
* & * \\
* & \hat{d}
\end{array}\right)\left(\begin{array}{ll}
\hat{u} & * \\
q & *
\end{array}\right) P_{1,0}(\mathbb{Z})
$$


where the indices run over

$$
(q, u, d) \in \coprod_{q \in \mathbb{N}: q \mid \ell}(\mathbb{Z} / \operatorname{gcd}(q, \ell / q) \mathbb{Z})^{\times} \times(\mathbb{Z} / \ell \mathbb{Z})^{\times} /\{ \pm 1\},
$$

where $\hat{u}, \hat{d}$ are prime to $\ell$ and give the class of $u \in(\mathbb{Z} / \operatorname{gcd}(q, \ell / q) \mathbb{Z})^{\times}$and $d \in$

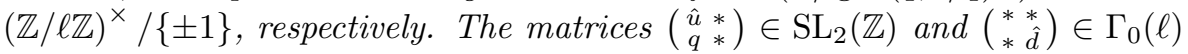
are completed in any manner. Furthermore, we may choose $\hat{d}$ and the upper left entry of $\left(\begin{array}{cc}* & * \\ * & \hat{d}\end{array}\right)\left(\begin{array}{cc}\hat{u} & * \\ q & *\end{array}\right)$ prime to any given natural number.

Proof. The double coset decomposition of $\tilde{\Gamma}_{1}(\ell)$ follows from the standard decomposition of $\Gamma_{1}(\ell)$ mentioned in the Introduction. Consider a representative

$$
\left(\begin{array}{ll}
a & b \\
c & \hat{d}
\end{array}\right)\left(\begin{array}{ll}
\hat{u} & * \\
q & *
\end{array}\right)=\left(\begin{array}{ll}
a \hat{u}+b q & * \\
c \hat{u}+\hat{d} q & *
\end{array}\right) .
$$

Note that $\operatorname{gcd}(a \hat{u}+b q, c \hat{u}+\hat{d} q)=1$. We will show that $\hat{d}$ and $a \hat{u}+b q$ may be chosen relatively prime to any given natural number without changing the double coset. Preserving $\operatorname{gcd}(\hat{d}, \ell)=1$, we may reselect $\hat{d}$ from the arithmetic progression $\hat{d}, \hat{d}+\ell, \ldots, \hat{d}+j \ell$, which contains infinitely many primes, and complete to a new matrix $\left(\begin{array}{ll}a & b \\ c & \hat{d}\end{array}\right) \in \Gamma_{0}(\ell)$. Consider this done. For $j \in \mathbb{N}$. the representative $\left(\begin{array}{ccc}a+j c & b+j \hat{d} \\ c & \hat{d}\end{array}\right) \in \Gamma_{0}(\ell)$ works just as well and the progression $(a+j c) \hat{u}+(b+j \hat{d}) q=(a \hat{u}+b q)+j(c \hat{u}+\hat{d} q)$ contains infinitely many primes because $a \hat{u}+b q$ and $c \hat{u}+\hat{d} q$ are relatively prime. Therefore we may pick both $\hat{d}$ and $a \hat{u}+b q$ prime to any fixed natural number.

\section{Equality among the $K(N) \mathcal{C}_{0}(\mathbb{Z}) P_{2,0}(\mathbb{Q})$}

We determine when representatives $\mathcal{C}_{0}(x)$ and $\mathcal{C}_{0}(y)$ give the same double coset in $K(N) \backslash \mathrm{Sp}_{2}(\mathbb{Q}) / P_{2,0}(\mathbb{Q})$.

Lemma 5.1. Let $N, m \in \mathbb{N}$ with $m \mid N$ and set $\ell=\operatorname{gcd}(m, N / m)$. Let $M \in \mathbb{Z}$ such that $\operatorname{gcd}(M, N)=1$. Then

$$
K(N) \mathcal{C}_{0}(M m) P_{2,0}(\mathbb{Q})=K(N) \mathcal{C}_{0}(M \ell) P_{2,0}(\mathbb{Q}) .
$$

Proof. Write $N=m \delta \ell$ and note $\ell \mid m$. Since $\operatorname{gcd}\left(\frac{m}{\ell}, \delta\right)=1$, there exist $a, z \in \mathbb{Z}$ such that $-a \frac{m}{\ell}+\delta z=1$. Since $\operatorname{gcd}(M, N)=1$, there exists $k \in \mathbb{Z}$ such that $M \mid(1+N k)$. Then define

$$
\begin{gathered}
P=\left(\begin{array}{cccc}
a \frac{m}{\ell}(1-\ell \delta M) & N \frac{m}{\ell}(M-k \ell \delta) & -\delta\left(m-\frac{1+N k}{M}\right) & a \delta \\
\frac{m}{\ell}(-M+k \ell) z & \frac{m}{\ell}(-1+\ell M \delta) & -\delta & \left(m-\frac{1+N k}{M}\right) \delta z / N \\
-M(1+m M-k N) z & N M^{2} & -(1+\ell M \delta) & (M-k \ell \delta) z \\
-N a M^{2} & -N M(-1-m M+k N) & N(-M+k \ell \delta) & a(1+M \ell \delta)
\end{array}\right), \\
Q=\left(\begin{array}{cccc}
-1-\ell \delta & \delta\left(m-\frac{1+N k}{M}\right) & \delta \\
\frac{z}{m} & a & -a \delta & -\left(m-\frac{1+N k}{M}\right) \delta z / N \\
0 & 0 & -1+\delta z & -\frac{z}{\ell} \\
0 & 0 & m \delta & -\frac{m}{\ell}
\end{array}\right) .
\end{gathered}
$$


We can see that $P \in K(N)$ and $Q \in P_{2,0}(\mathbb{Q})$. The result follows from directly checking that $P \mathcal{C}_{0}(M m) Q=\mathcal{C}_{0}(M \ell)$.

Lemma 5.2. Let $\ell, N \in \mathbb{N}$ with $\ell^{2} \mid N$. Let $M_{1}, M_{2} \in \mathbb{Z}$ such that $\operatorname{gcd}\left(M_{1}, N\right)$ $=\operatorname{gcd}\left(M_{2}, N\right)=1$. Then

$$
K(N) \mathcal{C}_{0}\left(M_{1} \ell\right) P_{2,0}(\mathbb{Q})=K(N) \mathcal{C}_{0}\left(M_{2} \ell\right) P_{2,0}(\mathbb{Q})
$$

if and only if $M_{1} \equiv \pm M_{2} \bmod \ell$.

Proof. Let $\delta=N / \ell^{2} \in \mathbb{N}$. Suppose that there exist $P \in K(N)$ and $Q \in P_{2,0}(\mathbb{Q})$ such that $P \mathcal{C}_{0}\left(M_{2} \ell\right) Q=\mathcal{C}_{0}\left(M_{1} \ell\right)$. By multiplying out the left hand side of $\mathcal{C}_{0}\left(M_{2} \ell\right)^{-1} P^{-1} \mathcal{C}_{0}\left(M_{1} \ell\right)=Q$, we see that, in order for $P$ to have paramodular divisibilities, $Q$ must be of the form

$$
Q=\left(\begin{array}{cccc}
a & b \ell & e & f \\
\frac{c}{\ell \delta} & d & g & h / N \\
0 & 0 & p & \frac{q}{\ell \delta} \\
0 & 0 & r \ell & s
\end{array}\right)
$$

where all variables are integers. We have

$$
Q J Q^{\prime}-J=\left(\begin{array}{cccc}
0 & \frac{-c e+b h-d f \ell \delta+a g \ell \delta}{\ell \delta} & \frac{b q+a p \delta}{\delta}-1 & \ell(a r+b s) \\
-\frac{-c e+b h-d f \ell \delta+a g \ell \delta}{\ell \delta} & 0 & \frac{c p+d q}{\ell \delta} & \frac{c r+d s \delta}{\delta}-1 \\
-\frac{b q+a p \delta}{\delta}+1 & -\frac{c p+d q}{\ell \delta} & 0 & 0 \\
-\ell(a r+b s) & -\frac{c r+d s \delta}{\delta}+1 & 0 & 0
\end{array}\right)
$$

and so the conditions for $Q$ to be symplectic are

$$
\begin{aligned}
-c e+b h-d f \ell \delta+a g \ell \delta & =0, \\
\frac{b q+a p \delta}{\delta} & =1, \\
a r+b s & =0, \\
c p+d q & =0, \\
\frac{c r+d s \delta}{\delta} & =1 .
\end{aligned}
$$

Now, $Q$ is symplectic if and only if $P$ is symplectic. We have

$$
\begin{aligned}
P^{-1} & =\mathcal{C}_{0}\left(M_{2} \ell\right) Q \mathcal{C}_{0}\left(M_{1} \ell\right)^{-1} \\
& =\left(\begin{array}{cccc}
a-f \ell M_{1} & \ell\left(b-e M_{1}\right) & e & f \\
\frac{c-h M_{1}}{\ell \delta} & d-g \ell M_{1} & g & h / N \\
\frac{c M_{2}-h M_{1} M_{2}-M_{1} q}{\delta} & \ell\left(d M_{2}-g \ell M_{1} M_{2}-M_{1} p\right) & g \ell M_{2}+p & \frac{h M_{2}+q}{\ell \delta} \\
\ell\left(a M_{2}-f \ell M_{1} M_{2}-s M_{1}\right) & \ell^{2}\left(b M_{2}-e M_{1} M_{2}-r M_{1}\right) & \ell\left(e M_{2}+r\right) & f \ell M_{2}+s
\end{array}\right),
\end{aligned}
$$


and so along with the symplectic conditions of $(2), P \in K(N)$ if and only if

$$
\begin{aligned}
& \ell \delta \mid b-e M_{1}, \\
& \ell \delta \mid c-h M_{1}, \\
& \delta \mid c M_{2}-h M_{1} M_{2}-q M_{1}, \\
& \ell \delta \mid d M_{2}-g \ell M_{1} M_{2}-M_{1} p, \\
& \ell \delta \mid h M_{2}+q, \\
& \ell \delta \mid a M_{2}-f \ell M_{1} M_{2}-s M_{1}, \\
& \delta \mid b M_{2}-e M_{2} M_{2}-r M_{1}, \\
& \ell \delta \mid e M_{2}+r .
\end{aligned}
$$

Thus we have that $\mathcal{C}_{0}\left(M_{1} \ell\right), \mathcal{C}_{0}\left(M_{2} \ell\right)$ are in the same double coset if and only if we can find integer solutions $a, b, c, d, e, f, g, h, p, q, r$ and $s$ to Equations (3) and (2) for given natural numbers $N, \ell$ and $\delta$ with $N=\ell^{2} \delta$ and given integers $M_{1}$ and $M_{2}$ prime to $N$.

First, suppose $\mathcal{C}_{0}\left(M_{1} \ell\right), \mathcal{C}_{0}\left(M_{2} \ell\right)$ are in the same double coset. It is not hard to see that Equations (3) imply that $\delta|c, \delta| b, \delta|q, \delta| r$. The last four equations in (2) imply that $d s-a p=d s(a p+b q / \delta)-a p(d s+c r / \delta)=$ $(d s b q-a p c r) / \delta=0$ because $d s b q=(d q)(b s)=(-c p)(-a r)=$ apcr. Thus $d s=a p$. This implies $b q=c r$. Then, noting that $s$ and $r$ cannot both be zero,

$$
\frac{a}{s}=\frac{d}{p}=\frac{-b / \delta}{r / \delta}=\frac{-c / \delta}{q / \delta} .
$$

Call this common value $\frac{u}{w}$ where $u, w \in \mathbb{Z}$ and $\operatorname{gcd}(u, w)=1$. Then for some $A, B, v, z \in Z$,

$$
\begin{aligned}
& a=A u, s=A w, d=B u, p=B w, \\
& b=-\delta v u, r=\delta v w, c=-\delta z u, q=\delta z w .
\end{aligned}
$$

The condition $\frac{b q+a p \delta}{\delta}=1$ becomes $-\delta v u z w+A u B w=1$, or

$$
u w(A B-\delta v z)=1 .
$$

This already implies that $u, w$ must be \pm 1 .

From Equations (3), we see $\ell \delta$ divides $M_{2}\left(b-e M_{1}\right)+M_{1}\left(e M_{2}+r\right)=$ $M_{2} b+M_{1} r=\left(-M_{2} \delta u v+M_{1} \delta w v\right)=\delta v\left(M_{1} w-M_{2} u\right)$. Hence

$$
\ell \mid\left(M_{1} w-M_{2} u\right) v .
$$

We similarly show that $\ell \mid\left(M_{1} w-M_{2} u\right) A$. From $\ell \delta \mid\left(a M_{2}-f \ell M_{1} M_{2}-s M_{1}\right)$, we get that $\ell$ divides $a M_{2}-s M_{1}=M_{2} A u-M_{1} A w=-\left(M_{1} w-M_{2} u\right) A$. Let $\lambda=\ell / \operatorname{gcd}\left(\ell, M_{1} w-M_{2} u\right)$, then it follows that $\lambda \mid A$ and $\lambda \mid v$. Then $\lambda \mid(A B-\delta v z)$ forces $\lambda=1$ and thus

$$
M_{1} w-M_{2} u \equiv 0 \bmod \ell .
$$

Since $u, w= \pm 1$, then we have

$$
M_{1} \equiv \pm M_{2} \quad \bmod \ell,
$$


as claimed.

On the other hand, suppose $M_{1} \equiv \pm M_{2} \bmod \ell$. Fix $u, w \in\{ \pm 1\}$ such that $M_{1} w-M_{2} u=\ell y$ for some $y \in \mathbb{Z}$. Let $t_{1}, t_{2} \in \mathbb{Z}$ such that $N \mid\left(t_{1} M_{1}-1\right)$ and $N \mid\left(t_{2} M_{2}-1\right)$. Let

$$
\begin{gathered}
P=\left(\begin{array}{cccc}
u w\left(u+\ell M_{1} t_{1} t_{2} y\right) & 0 & 0 & -u w t_{1} t_{2} y \\
0 & u+\ell M_{1} t_{1} t_{2} y & -t_{1} t_{2} y & 0 \\
0 & N y M_{1} M_{2} t_{2} t_{2}-1 & w-\ell M_{2} t_{1} t_{2} y & 0 \\
N u w y \frac{M_{1} M_{2} t_{1} t_{2}-1}{\delta} & 0 & 0 & u w\left(w-\ell M_{2} t_{1} t_{2} y\right)
\end{array}\right), \\
Q=\left(\begin{array}{cccc}
w & 0 & 0 & t_{1} t_{2} y \\
0 & u & u w t_{1} t_{2} y & 0 \\
0 & 0 & w & 0 \\
0 & 0 & 0 & u
\end{array}\right) .
\end{gathered}
$$

Then $P \in K(N)$ and $Q \in P_{2,0}(\mathbb{Q})$ and

$$
P \mathcal{C}_{0}\left(M_{1} \ell\right) Q=\mathcal{C}_{0}\left(M_{2} \ell\right)
$$

can be verified directly.

Proposition 5.3. Let $N \in \mathbb{N}$. Let $\ell_{1}^{2}, \ell_{2}^{2} \mid N$ with $\ell_{1}, \ell_{2} \in \mathbb{N}$. Let $M_{1}, M_{2} \in \mathbb{Z}$ such that $\operatorname{gcd}\left(M_{1}, N\right)=\operatorname{gcd}\left(M_{2}, N\right)=1$. Then

$$
K(N) \mathcal{C}_{0}\left(M_{1} \ell_{1}\right) P_{2,0}(\mathbb{Q})=K(N) \mathcal{C}_{0}\left(M_{2} \ell_{2}\right) P_{2,0}(\mathbb{Q})
$$

if and only if $\ell_{1}=\ell_{2}$ and $M_{1} \equiv \pm M_{2} \bmod \ell_{1}$.

Proof. Since $\ell_{1}^{2}, \ell_{2}^{2} \mid N$, let $\delta=\frac{N}{\ell_{1} \ell_{2}} \in \mathbb{N}$. In light of Lemma 5.2, we only need to prove that $K(N) \mathcal{C}_{0}\left(M_{1} \ell_{1}\right) P_{2,0}(\mathbb{Q})=K(N) \mathcal{C}_{0}\left(M_{2} \ell_{2}\right) P_{2,0}(\mathbb{Q})$ implies $\ell_{1}=\ell_{2}$. So assume there exist $P \in K(N)$ and $Q \in P_{2,0}(\mathbb{Q})$ such that $P \mathcal{C}_{0}\left(M_{2} \ell_{2}\right) Q=$ $\mathcal{C}_{0}\left(M_{1} \ell_{1}\right)$. Since $\mathcal{C}_{0}\left(M_{2} \ell_{2}\right)^{-1} P^{-1} \mathcal{C}_{0}\left(M_{1} \ell_{1}\right)=Q$, by multiplying out the left hand side, we see that $Q$ must be of the form

$$
Q=\left(\begin{array}{cccc}
a & b \ell_{1} & e & f \\
\frac{c}{\ell_{2} \delta} & d & g & h / N \\
0 & 0 & p & \frac{q}{\ell_{1} \delta} \\
0 & 0 & r \ell_{2} & s
\end{array}\right)
$$

where all variables are integers. We have

$$
Q J Q^{\prime}-J=\left(\begin{array}{cccc}
0 & * & a p+\frac{b q}{\delta}-1 & * \\
* & 0 & * & * \\
* & * & 0 & 0 \\
* & * & 0 & 0
\end{array}\right),
$$

and so in particular, we must have

$$
a p+\frac{b q}{\delta}=1
$$

Now, we have

$$
P^{-1}=\mathcal{C}_{0}\left(M_{2} \ell_{2}\right) Q \mathcal{C}_{0}\left(M_{1} \ell_{1}\right)^{-1}
$$




$$
=\left(\begin{array}{cccc}
a-f \ell_{1} M_{1} & \ell_{1}\left(b-e M_{1}\right) & e & f \\
\frac{c-h M_{1}}{\ell_{2} \delta} & d-g \ell_{1} M_{1} & g & h / N \\
\frac{c M_{2}-h M_{1} M_{2}-M_{1} q}{\delta} & \ell_{2} d M_{2}-g \ell_{1} \ell_{2} M_{1} M_{2}-\ell_{1} M_{1} p & g \ell_{2} M_{2}+p & \frac{h M_{2}+q}{\ell_{1} \delta} \\
\ell_{2} a M_{2}-f \ell_{1} \ell_{2} M_{1} M_{2}-s \ell_{1} M_{1} & \ell_{1} \ell_{2}\left(b M_{2}-e M_{1} M_{2}-r M_{1}\right) & \ell_{2}\left(e M_{2}+r\right) & f \ell_{2} M_{2}+s
\end{array}\right) .
$$

The divisibility conditions of $P^{-1} \in K(N)$ force that $\delta|c, \delta| b, \delta|q, \delta| r$. Let $b=\delta b^{\prime}$ and $q=\delta q^{\prime}$. Now let $v=\operatorname{gcd}\left(\ell_{1}, \ell_{2}\right)$ and denote $\ell_{1}^{\prime}=\ell_{1} / v$ and $\ell_{2}^{\prime}=\ell_{2} / v$ so that $\operatorname{gcd}\left(\ell_{1}^{\prime}, \ell_{2}^{\prime}\right)=1$. Then the condition that

$$
N \mid\left(\ell_{2} a M_{2}-f \ell_{1} \ell_{2} M_{1} M_{2}-s \ell_{1} M_{1}\right)
$$

implies that $\ell_{1} \mid \ell_{2} a M_{2}$, which implies $\ell_{1} \mid \ell_{2} a$, which implies $\ell_{1}^{\prime} \mid \ell_{2}^{\prime} a$. Then $\ell_{1}^{\prime} \mid a$. Let $a=\ell_{1}^{\prime} a^{\prime}$. Then $a p+\frac{b q}{\delta}=1$ can be rewritten as

$$
a^{\prime} \ell_{1}^{\prime} p+\delta b^{\prime} q^{\prime}=1 .
$$

But $N=\ell_{1} \ell_{2} \delta$ and $\ell_{1}^{2} \mid N$ implies that $\ell_{1} \mid \ell_{2} \delta$ which implies $\ell_{1}^{\prime} \mid \delta$. Then Equation (4) implies $\ell_{1}^{\prime} \mid 1$, which forces $\ell_{1}^{\prime}=1$. This means $\ell_{1} \mid \ell_{2}$. By the same argument, we must also have $\ell_{2} \mid \ell_{1}$. Thus $\ell_{1}=\ell_{2}$.

\section{Reduction of the zero-cusps to canonical form}

In this section we show that all double cosets $K(N) \sigma P_{2,0}(\mathbb{Q})$, for $\sigma \in$ $S p_{2}(\mathbb{Q})$, are of the form $K(N) \mathcal{C}_{0}(x) P_{2,0}(\mathbb{Q})$ for some $x \in \mathbb{Z}$. We begin the reduction to this canonical form by relying on the classification of the onecusps. From $\sigma \in K(N) u\left(\begin{array}{cc}1 & m \\ 0 & 1\end{array}\right) P_{2,1}(\mathbb{Q})$ for some $m \in \mathbb{Z}$, it follows that $\sigma \in$ $K(N) u\left(\begin{array}{cc}1 & m \\ 0 & 1\end{array}\right) i_{2,1}(\kappa) P_{2,0}(\mathbb{Q})$ for some $\kappa \in \mathrm{SL}_{2}(\mathbb{Z})$.

Lemma 6.1. Let $N, m \in \mathbb{N}$ with $m \mid N$. For all $\left(\begin{array}{ll}a & b \\ c & d\end{array}\right) \in S L_{2}(\mathbb{Z})$,

$$
K(N) \mathcal{C}_{1}(m) i_{2,1}\left(\begin{array}{ll}
a & b \\
c & d
\end{array}\right) P_{2,0}(\mathbb{Q})=K(N)\left(\begin{array}{cccc}
1 & 0 & 0 & 0 \\
0 & 1 & 0 & 0 \\
0 & m c & 1 & 0 \\
m c & m^{2} c d & 0 & 1
\end{array}\right) P_{2,0}(\mathbb{Q}) .
$$

Proof. The result follows, using $a d-b c=1$, from the calculation:

$$
\begin{aligned}
\mathcal{C}_{1}(m) i_{2,1}\left(\begin{array}{ll}
a & b \\
c & d
\end{array}\right) & =\left(\begin{array}{cccc}
a & m & b & 0 \\
0 & 1 & 0 & 0 \\
c & 0 & d & 0 \\
-c m & 0 & -d m & 1
\end{array}\right) \\
& =\left(\begin{array}{cccc}
-a & 0 & -b & 0 \\
0 & 1 & 0 & 0 \\
-c & 0 & -d & 0 \\
0 & 0 & 0 & 1
\end{array}\right)\left(\begin{array}{cccc}
1 & 0 & 0 & 0 \\
0 & 1 & 0 & 0 \\
0 & m c & 1 & 0 \\
m c & m^{2} c d & 0 & 1
\end{array}\right)\left(\begin{array}{cccc}
-1 & -d m & 0 & 0 \\
0 & 1 & 0 & 0 \\
0 & 0 & -1 & 0 \\
0 & 0 & -d m & 1
\end{array}\right) .
\end{aligned}
$$

We now show that the $\mathcal{C}_{0}(\mathbb{Z})$ give double coset representatives for the zerocusps $K(N) \backslash \mathcal{H}_{0}^{*}$ by tracking the zero-cusps of the boundary curves $K(N) \backslash \mathcal{H}_{1}^{*}$.

Theorem 6.2. Let $N \in \mathbb{N}$ and $\sigma \in \mathrm{Sp}_{2}(\mathbb{Q})$. There are $R, r \in \mathbb{N}$ with $r \mid N$ and $R$ relatively prime to $N$ such that $K(N) \sigma P_{2,0}(\mathbb{Q})=K(N) \mathcal{C}_{0}(R r) P_{2,0}(\mathbb{Q})$.

Furthermore for $m \in \mathbb{N}$ with $m \mid N$, set $\ell=\operatorname{gcd}(m, N / m)$ and $\delta=N /(m \ell)$ so that $N=m \ell \delta$. Let $\tilde{\Gamma}_{1}(\ell)\left(\begin{array}{c}* * \\ * \\ *\end{array}\right)\left(\begin{array}{c}\hat{u} * \\ q *\end{array}\right) P_{1,0}(\mathbb{Z})$ be a double coset from

$$
\tilde{\Gamma}_{1}(\ell) \backslash \mathrm{SL}_{2}(\mathbb{Z}) / P_{1,0}(\mathbb{Z})
$$


chosen as in Lemma 4.4 with: $\left(\begin{array}{l}* * \\ * \\ *\end{array}\right) \in \Gamma_{0}(\ell),\left(\begin{array}{c}\hat{u} * \\ q *\end{array}\right) \in \mathrm{SL}_{2}(\mathbb{Z}), q \mid \ell$ and $\hat{d}$ and the upper left entry of $\left(\begin{array}{c}* * \\ * d\end{array}\right)\left(\begin{array}{c}\hat{u} \\ q\end{array} * \begin{array}{c}* \\ q\end{array}\right)$ relatively prime to $N$. Set

$$
D=\left(\begin{array}{ll}
1 & 0 \\
0 & \delta
\end{array}\right)\left(\begin{array}{ll}
* & * \\
* & \hat{d}
\end{array}\right)\left(\begin{array}{cc}
\hat{u} & * \\
q & *
\end{array}\right)\left(\begin{array}{cc}
1 & 0 \\
0 & \delta
\end{array}\right)^{-1}
$$

so that the corresponding double coset from $G(\ell, \delta) \backslash \mathrm{SL}_{2}(\mathbb{Q}) / P_{1,0}(\mathbb{Q})$ is $G(\ell, \delta) D P_{1,0}(\mathbb{Q})$. We have $i_{2,1}(D) \in K(N) \mathcal{C}_{0}((\ell / q) \hat{d}) P_{2,0}(\mathbb{Q})$ with $(\ell / q) \mid N$ and $\hat{d}$ relatively prime to $N$.

Proof. By Theorem 1.1, we can write $\sigma \in K(N) \mathcal{C}_{1}(m) P_{2,1}(\mathbb{Q})$ for some $m \in \mathbb{N}$ with $m \mid N$. Thus $\sigma \in K(N) \mathcal{C}_{1}(m) i_{2,1}\left(\begin{array}{ll}a & b \\ c & d\end{array}\right) P_{2,0}(\mathbb{Q})$ for some $\left(\begin{array}{ll}a & b \\ c & d\end{array}\right) \in \mathrm{SL}_{2}(\mathbb{Q})$. This double coset is unchanged if we reselect $\left(\begin{array}{ll}a & b \\ c & d\end{array}\right)$ from $G(\ell, \delta)\left(\begin{array}{ll}a & b \\ c & d\end{array}\right) P_{1,0}(\mathbb{Q})$. This is not altogether trivial; it uses the normality of the Heisenberg subgroup $H_{2,1}$ in $P_{2,1}(\mathbb{Q})$.

As in the statement of this theorem, first select

$$
D=\left(\begin{array}{cc}
1 & 0 \\
0 & \delta
\end{array}\right)\left(\begin{array}{cc}
* * \\
* & *
\end{array}\right)\left(\begin{array}{cc}
\hat{u} & * \\
q & *
\end{array}\right)\left(\begin{array}{cc}
1 & 0 \\
0 & \delta
\end{array}\right)^{-1}=\left(\begin{array}{cc}
1 & 0 \\
0 & \delta
\end{array}\right)\left(\begin{array}{cc}
w & * \\
z & *
\end{array}\right)\left(\begin{array}{cc}
1 & 0 \\
0 & \delta
\end{array}\right)^{-1}=\left(\begin{array}{cc}
w & * \\
\delta & *
\end{array}\right)
$$

for some integer $z \equiv \hat{d} q \bmod \ell$ with $\operatorname{gcd}(\hat{d}, N)=1$ and for some integer $w$ with $\operatorname{gcd}(w, N)=1$. It follows that $\operatorname{gcd}(w, \delta z)=1$. Therefore, we can multiply $D$ on the right by something in $P_{1,0}(\mathbb{Q})$ to obtain $D^{\prime}=\left(\begin{array}{c}w \\ \delta z\end{array}\right) \in S L_{2}(\mathbb{Z})$ and $D^{\prime}$ is in the same double coset as $D$. By Lemma 6.1 , we have the equality of double cosets:

$$
K(N) \mathcal{C}_{1}(m) i_{2,1}\left(D^{\prime}\right) P_{2,0}(\mathbb{Q})=K(N)\left(\begin{array}{cccc}
1 & 0 & 0 & 0 \\
0 & 1 & 0 & 0 \\
0 & m \delta z & 1 & 0 \\
m \delta z & m^{2} \delta z y & 0 & 1
\end{array}\right) P_{2,0}(\mathbb{Q}) .
$$

Next, we note that $N \mid \delta m^{2}$, so that the simple element $\left(\begin{array}{ll}I & 0 \\ S\end{array}\right)$ is in $K(N)$ for $S=$ $\left(\begin{array}{cc}0 & 0 \\ 0 & m^{2} \delta z y\end{array}\right)$. So we obtain $K(N) \mathcal{C}_{1}(m) i_{2,1}\left(D^{\prime}\right) P_{2,0}(\mathbb{Q})=K(N) \mathcal{C}_{0}(m \delta z) P_{2,0}(\mathbb{Q})$. We have $z=\hat{d} q+k \ell$ for some integer $k$, so that $m \delta z=m \delta \hat{d} q+k N$ and $K(N) \mathcal{C}_{0}(m \delta z) P_{2,0}(\mathbb{Q})=K(N) \mathcal{C}_{0}(m \delta q \hat{d}) P_{2,0}(\mathbb{Q})$. Using Lemma 5.1, and the equality $\operatorname{gcd}\left(m \delta q, \frac{N}{m \delta q}\right)=\ell / q$, which follows from $\ell \mid m$, we have

$$
K(N) \mathcal{C}_{0}(m \delta z) P_{2,0}(\mathbb{Q})=K(N) \mathcal{C}_{0}((\ell / q) \hat{d}) P_{2,0}(\mathbb{Q}),
$$

which is the final assertion of the theorem. The first assertion follows by setting $R=\hat{d}$ and $r=\ell / q$.

\section{Proofs}

We are now ready to prove Theorem 1.3 on the zero-cusps of the Satake compactification and Theorem 1.4 on the zero-dimensional cusps of the onedimensional cusps as stated in the Introduction.

Proof of Theorem 1.3. From Theorem 6.2, we know that

$$
\mathrm{Sp}_{2}(\mathbb{Q})=\bigcup_{r, R} K(N) \mathcal{C}_{0}(R r) P_{2,0}(\mathbb{Q})
$$


where $r$ runs over $r \mid N$ and $R$ is prime to $N$. This countable union however, is not disjoint. By Lemma 5.1, we know we can restrict the union to $r=\ell=$ $\operatorname{gcd}(m, N / m)$, that is, to $\ell \mid f$. By Lemma 5.2, we know that for fixed $\ell$ we can restrict $R$ to run over the classes of $(\mathbb{Z} / \ell \mathbb{Z})^{\times} /\{ \pm 1\}$. This union is then disjoint by Proposition 5.3. Counting the 0 -cusps has already been left as an exercise to the reader.

Proof of Theorem 1.4. Using the decomposition from Theorem 1.3, for each $b, c \in \mathbb{N}$ where $c^{2} \mid N$ and $\operatorname{gcd}(b, N)=1$, we need to count how many times a double coset $G(\ell, \delta) D P_{1,0}(\mathbb{Q})$ yields the double coset equality

$$
K(N) \mathcal{C}_{1}(m) i_{2,1}(D) P_{2,0}(\mathbb{Q})=K(N) \mathcal{C}_{0}(b c) P_{2,0}(\mathbb{Q}) .
$$

From Theorem 6.2 and by Proposition 5.3, and using the notation from the proof of Theorem 6.2 , we can see that each such $D$ must come from $\left(\begin{array}{cc}* & * \\ * & \hat{d}\end{array}\right)\left(\begin{array}{cc}\hat{u} & * \\ q & *\end{array}\right)$

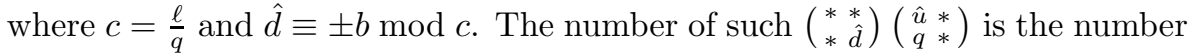
of $\left(\begin{array}{cc}\hat{u} & * \\ q & *\end{array}\right)$ times the number of $\operatorname{such}\left(\begin{array}{cc}* & * \\ * & \hat{d}\end{array}\right)$. The number of $\left(\begin{array}{ll}\hat{u} & * \\ q & *\end{array}\right)$ is $\varphi(\operatorname{gcd}(q, \ell / q))$. The number of $\left(\begin{array}{c}* \\ *\end{array}\right)$ with $\hat{d} \equiv \pm b \bmod c$ is the number of elements from $(\mathbb{Z} / \operatorname{lcm}(q, \ell / q) \mathbb{Z})^{\times} /( \pm 1)$ that are equivalent to $\pm b \bmod c$, which is $\frac{\varphi^{\prime}(\operatorname{lcm}(q, \ell / q))}{\varphi^{\prime}(c)}$. Plugging back in $q=\ell / c$ gives the formula $\frac{\varphi^{\prime}(\operatorname{lcm}(c, \ell / c)) \varphi(\operatorname{gcd}(c, \ell / c))}{\varphi^{\prime}(c)}$. The second formula follows by considering the cases of $c \leq 2$ and $c>2$, and noting that when $c \leq 2$ we have $\varphi(c)=1$ and $\varphi(\operatorname{lcm}(c, \ell / c))=\varphi(\ell / c)$.

Proposition 7.1. Let $N, N_{0}, f \in \mathbb{N}$ with $N=f^{2} N_{0}$ and $N_{0}$ squarefree. For even $k>4$ we have

$$
\begin{aligned}
& \operatorname{dim} M_{k}(K(N))-\operatorname{dim} S_{k}(K(N)) \\
= & 1+\llcorner f / 2\lrcorner+\sum_{m \in \mathbb{N}: m \mid N} \operatorname{dim} S_{k}\left(\tilde{\Gamma}_{1}(\operatorname{gcd}(m, N / m))\right) .
\end{aligned}
$$

Proof. In the paramodular case by Theorem $4.3, \Gamma_{m}=G(\ell, \delta) \cong \tilde{\Gamma}_{1}(\ell)$ for $\ell=\operatorname{gcd}(m, N / m)$ and $N=m \ell \delta$. For even $k>4$, by Satake's theorem [18], the codimension of the cusp forms is the dimension of the modular forms in $\oplus_{m \mid N} M_{k}\left(\Gamma_{m}\right)$ that satisfy condition (1) of section 3. All the cusp forms $\oplus_{m \mid N} S_{k}\left(\Gamma_{m}\right)$ satisfy condition (1). The dimension of the Eisenstein series satisfying condition (1) is the number of zero-cusps of $\mathcal{S}\left(K(N) \backslash \mathcal{H}_{2}\right)$ because, in the elliptic modular case for even $k \geq 4$, there is a basis of Eisenstein series supported at single cusps, compare the dimension formulae in [4], pages 87-88.

\section{Appendix}

Proposition 4.1 (Gritsenko). Let $N \in \mathbb{N}$ and $[X] \in \mathbb{P}^{3}(\mathbb{Q})$. We have $K(N)[X]=K(N)\left[\left(\begin{array}{llll}d & 1 & 0 & 0\end{array}\right)^{\prime}\right]$ for $1-\operatorname{inv}(X)=d \mathbb{Z} \supseteq N \mathbb{Z}$. 
Proof. We may select a representative $X \in L_{N}$ with $X^{\prime} L_{N}^{*}=\mathbb{Z}$. In coordinate form, writing $X^{\prime}=\left(x_{1}, x_{2}, x_{3}, x_{4}\right)$, we may assume that $x_{1}, x_{2}, x_{3}, x_{4} / N$ are integral and that $\operatorname{gcd}\left(x_{1}, x_{2}, x_{3}, x_{4} / N\right)=1$. Both these properties are preserved by the action of the paramodular group $K(N)$. First, we select a representative $X$ with $x_{4}=0$ by the following procedure: For $X \in L_{N}$, if $x_{4} \neq 0$ then $g=\operatorname{gcd}\left(x_{2}, x_{4} / N\right)$ is also nonzero and we have $g=a x_{2}+b x_{4} / N$ for some integers $a$ and $b$. We obtain

$$
\left(\begin{array}{cccc}
1 & 0 & 0 & 0 \\
0 & a & 0 & b / N \\
0 & 0 & 1 & 0 \\
0 & -\left(\frac{x_{4}}{g N}\right) N & 0 & \frac{x_{2}}{g}
\end{array}\right)\left(\begin{array}{c}
x_{1} \\
x_{2} \\
x_{3} \\
x_{4}
\end{array}\right)=\left(\begin{array}{c}
x_{1} \\
g \\
x_{3} \\
0
\end{array}\right) \in K(N) X
$$

Second, we select a representative $X$ with $x_{3}=x_{4}=0$ : take $\left(\begin{array}{ll}a & b \\ c & d\end{array}\right) \in S L_{2}(\mathbb{Z})$ with $\left(\begin{array}{ll}a & b \\ c & d\end{array}\right)\left(\begin{array}{l}x_{1} \\ x_{3}\end{array}\right)=\left(\begin{array}{c}m \\ 0\end{array}\right)$ for $m=\operatorname{gcd}\left(x_{1}, x_{3}\right)$ so that

$$
\left(\begin{array}{llll}
a & 0 & b & 0 \\
0 & 1 & 0 & 0 \\
c & 0 & d & 0 \\
0 & 0 & 0 & 1
\end{array}\right)\left(\begin{array}{c}
x_{1} \\
x_{2} \\
x_{3} \\
0
\end{array}\right)=\left(\begin{array}{c}
m \\
x_{2} \\
0 \\
0
\end{array}\right)
$$

Third, we select a representative $X$ with $x_{4}=N$. Since $X^{\prime} L_{N}^{*}=\mathbb{Z}$, we have $\operatorname{gcd}\left(m, x_{2}\right)=1$ and there are integers $a$ and $b$ with $a m+b x_{2}=1$, so that

$$
\left(\begin{array}{cccc}
1 & 0 & 0 & 0 \\
0 & 1 & 0 & 0 \\
0 & a N & 1 & 0 \\
a N & b N & 0 & 1
\end{array}\right)\left(\begin{array}{c}
m \\
x_{2} \\
0 \\
0
\end{array}\right)=\left(\begin{array}{c}
m \\
x_{2} \\
a N x_{2} \\
N
\end{array}\right)
$$

Fourth, we get a representative with $x_{2}=1$ and $x_{4}=0$. Since $N \neq 0$, we may apply the procedure of the first step as before but note that this time $g=$ $\operatorname{gcd}\left(x_{2}, 1\right)=1$, so that we have a representative of the form $X^{\prime}=\left(x_{1}, 1, x_{3}, 0\right)$. Fifth, by a repetition of the second step we have a representative of the form $X^{\prime}=(m, 1,0,0)$. Finally, notice that $1-\operatorname{inv}(X)=m \mathbb{Z}+N \mathbb{Z}=d \mathbb{Z}$ so that $\alpha m+\beta N=d$ for some integers $\alpha$ and $\beta$. We have Gritsenko's result:

$$
\left(\begin{array}{cccc}
0 & 0 & 1 & 0 \\
0 & 1 & 0 & 0 \\
-1 & 0 & 0 & 0 \\
0 & 0 & 0 & 1
\end{array}\right)\left(\begin{array}{cccc}
1 & 0 & -\frac{m}{d} & 0 \\
0 & 1 & 0 & 0 \\
0 & 0 & 1 & 0 \\
0 & 0 & 0 & 1
\end{array}\right)\left(\begin{array}{cccc}
1 & 0 & 0 & 0 \\
0 & 1 & 0 & 0 \\
\alpha & \beta N & 1 & 0 \\
\beta N & -\beta m N & 0 & 1
\end{array}\right)\left(\begin{array}{c}
m \\
1 \\
0 \\
0
\end{array}\right)=\left(\begin{array}{l}
d \\
1 \\
0 \\
0
\end{array}\right) \in K(N) X .
$$

\section{References}

[1] S. Böcherer and T. Ibukiyama, Surjectivity of Siegel $\Phi$-operator for square free level and small weight, Annales de l'institut Fourier 62 (2012), no. 1, 121-144.

[2] A. Brumer and K. Kramer, Paramodular abelian varieties of odd conductor, arXiv: 1004.4699

[3] U. Christian, Einführung in die Theorie der paramodularen Gruppen, Math. Ann. 168 (1967), 59-104.

[4] F. Diamond and J. Shurman, A First Course in Modular Forms, Graduate Texts in Mathematics, 228. Springer-Verlag, New York, 2005. xvi+436 pp. 
[5] E. Freitag, Siegelsche Modulfunktionen, Grundlehren der mathematischen Wissenschaften, Band 254, Berlin-Heidelberg-New York, Springer-Verlag, 1983.

[6] V. Gritsenko, Irrationality of the moduli spaces of polarized abelian surfaces, Internat. Math. Res. Notices 1994 (1994), no. 6, 235 ff., approx. 9 pp.

[7] V. Gritsenko and K. Hulek, Minimal Siegel modular threefolds, Math. Proc. Cambridge Philos. Soc. 123 (1998), no. 3, 461-485.

[8] T. Ibukiyama, On some alternating sum of dimensions of Siegel cusp forms of general degree and cusp configurations, J. Fac. Sci. Univ. Tokyo Sec. IA Math. 40 (1993), no. $2,245-283$.

[9] _ Dimension formulas of Siegel modular forms of weight 3 and supersingular abelian surfaces, Siegel Modular Forms and Abelian Varieties, Proceedings of the 4-th Spring Conference on Modular Forms and Related Topics (2007), 39-60.

[10] - On relations of dimensions of automorphic forms of $\operatorname{Sp}(2, \mathbf{R})$ and its compact twist $S p(2)$ (I), Automorphic forms and number theory (Sendai, 1983), 7-30, Adv. Stud. Pure Math., 7, North-Holland, Amsterdam, 1985.

[11] T. Ibukiyama and H. Kitayama, Dimensions of paramodular cusp forms for squarefree levels, preprint.

[12] T. Ibukiyama, C. Poor, and D. Yuen, Jacobi forms that characterize paramodular forms, preprint, 2011.

[13] J.-I. Igusa, Theta Functions, Die Grundlehren der mathematischen Wissenschaften, Band 194, Springer-Verlag, New York-Heidelberg, 1972.

[14] H. Reefschläger, Berechnung der Anzahl der 1-Spitzen der Paramodularen Gruppen 2ten Grades, Dissertation, Georg-August-Universität zu Göttingen, 1973.

[15] B. Roberts and R. Schmidt, Local Newforms for GSp(4), Springer Lecture Note in Mathematics, vol. 1918, Springer-Verlag, 2007.

[16] I. Satake, Compactification de espaces quotients de Siegel II, Séminaire Cartan, E. N. S., 1957/58, Exposeé 13, 1-10.

[17] _ L'opérateur $\Phi$, Séminaire Cartan, E. N. S., 1957/58, Exposeé 14, 1-18.

[18] _ Surjectivité globale de opérateur $\Phi$, Séminaire Cartan, E. N. S., 1957/58, Exposeé $16,1-17$.

[19] C. Siegel, Moduln Abelscher Funktionen, Nachrichten der Akademie der Wissenschaften in Göttingen, Mathematisch-physikalische Klasse. 25 (1960), 365-427.

CRIS POOR

Department of Mathematics

FordHAM UNIVERSITY

BRONX, NY 10458, USA

E-mail address: poor@fordham.edu

DAVID S. YUEN

Department of Mathematics and Computer Science

Lake Forest College

555 N. Sheridan Rd., Lake Forest, IL 60045, USA

E-mail address: yuen@lakeforest.edu 